\title{
Spontaneous Symmetry Breaking through Mixing
}

\author{
Harald Fritzsch and Michael Spannowsky \\ Ludwig Maximilian University \\ Sektion Physik, Theresienstr. 37, 80333 München Germany
}

\begin{abstract}
:
We discuss a model, in which the negative mass square needed in the Higgs mechanism is generated by mixing with a heavy scalar. We have two scalar doublets in the standard model. Phenomenological properties of the heavy new scalar are discussed. The heavy scalar can be detected by the LHC.

In the Standard Model (SM) the masses of the W- and Z-bosons and of the fermions (leptons and quarks) are introduced by the mechanism of spontaneous symmetry breaking ("Higgs mechanism"). It is assumed that the squared mass term of the scalar particle is negative. This, together with the $\phi^{4}$ self interaction of the scalar bosons leads to a non-zero vacuum expectation value.

The masses of the W- and Z-bosons are spontaneously generated via the gauge interaction, and the masses of the fermions appear due to their Yukawa-type interactions with the scalar. No prediction can be made about the fermion masses, since they depend on the Yukawa coupling constants, which are free parameters.

The negative mass square of the scalar boson is rather peculiar and looks to us like an ad-hoc assumption, not explaining why the electroweak gauge symmetry is broken. In this paper we would like to propose a possible underlying mechanism, able to generate the imaginary Higgs mass (see also [1]) at an energy scale testable at the LHC.

We assume that there is a massless scalar which mixes (seesaw like) with a heavy scalar. Through the mixing a negative mass for the scalar is introduced in a natural way. As a result of the mixing the spontaneous symmetry breaking arises as in the Standard Model. For a long time, the seesaw mechanism has been widely studied to generate light neutrino masses 2] as well as to explain the mass hierarchies in the whole fermion sector 3. Instead, the idea of applying this mechanism to the scalar sector of the SM beside incorporating new degrees of freedom is quite new and recieved increasing interest over the last years 4 .
\end{abstract}

The scalar sector of this model is given by the following Lagrange density:

$$
L_{s c .}=D^{\mu}\left(\begin{array}{c}
\phi \\
\Phi
\end{array}\right)^{+} D_{\mu}\left(\begin{array}{c}
\phi \\
\Phi
\end{array}\right)-\left(\lambda \phi^{+} \Phi+\lambda \Phi^{+} \phi+\Lambda \Phi^{2}-\kappa\left(\phi^{+} \phi\right)^{2}\right)
$$

The mass matrix is given by

$$
M=\left(\begin{array}{cc}
0 & \lambda \\
\lambda & \Lambda
\end{array}\right)
$$

where we assume $\lambda \ll \Lambda$.

We diagonalize this mass matrix using

$$
S=\left(\begin{array}{cc}
\cos \theta & -\sin \theta \\
\sin \theta & \cos \theta
\end{array}\right)
$$

The mixing angle $\theta$ is fixed by $\lambda$ and $\Lambda$.

$$
\begin{aligned}
& M^{\prime}=S M S^{-1} \\
& M^{\prime}=\left(\begin{array}{cc}
1 / 2\left(\Lambda-\sqrt{4 \lambda^{2}+\Lambda^{2}}\right) & 0 \\
0 & 1 / 2\left(\Lambda+\sqrt{4 \lambda^{2}+\Lambda^{2}}\right)
\end{array}\right)
\end{aligned}
$$


The assumption $\lambda \ll \Lambda$ ensures that $|\sin \theta| \ll|\cos \theta|$.

Note that the negative mass squared for the light scalar is proportional to $\lambda^{2}$ in first order.

$$
\begin{aligned}
& \mu^{2}(\lambda, \Lambda) \equiv-1 / 2\left(\Lambda-\sqrt{4 \lambda^{2}+\Lambda^{2}}\right) \\
& \rho^{2}(\lambda, \Lambda) \equiv 1 / 2\left(\Lambda+\sqrt{4 \lambda^{2}+\Lambda^{2}}\right)
\end{aligned}
$$

where $\mu^{2}, \rho^{2}>0$.

Beside the light scalar there is a heavy scalar $\Phi$, which is an isospin doublet. As far as the light scalar is concerned, it acts like the Higgs particle in the Standard Model. Besides this particle there is, however, the new heavy scalar

$$
\Phi^{\prime}=\left(\begin{array}{c}
H^{+} \\
H_{0}+i H_{1}
\end{array}\right) .
$$

with $\phi=\phi^{\prime} \cos \theta-\Phi^{\prime} \sin \theta$.

$S$ is, of course, an orthogonal transformation. Thus the purely kinetic part of the lagrangian is not altered. There is no need to introduce for this particle a self-interaction proportional to the field in the fourth power or any Yukawa couplings. But because of the $\phi^{4}$ term in the scalar potential and the Yukawa coupling of $\phi$ there are couplings between the $\Phi^{\prime}$ and the $\phi^{\prime}$ as well as Yukawa like couplings for the heavy scalar, induced by the mixing. These new interactions depend on the ratio $\lambda / \Lambda$. The larger the mass difference between those particles, the more are non-standard-model couplings suppressed in the Yukawa and scalar sector.

The minimum of the Higgs potential is given by

$$
\langle\phi\rangle=\sqrt{\frac{\mu^{2}}{2 \kappa \cos \theta}}
$$

and we have the tree-level relations

$$
M_{h}=\sqrt{2} \mu
$$

and

$$
M_{H}=\left(3 M_{h}^{2} \tan ^{2} \theta+2 \rho^{2}\right)^{1 / 2}
$$

If the masses of the scalars were much heavier than the Higgs boson mass, the mixing between those particles would be strongly suppressed and could be neglected. For example, if the Higgs mass is $M_{h}=$ $116 \mathrm{GeV}$ and the new scalars mass is $M_{\phi^{\prime}} \approx 16 \mathrm{TeV}$, these couplings are suppressed by powers of the factor $|\sin \theta| \approx 0.008$.

Neglecting the mixing terms it follows from $\left\langle\Phi^{\prime}\right\rangle=0$ that there are just four particle couplings between gauge bosons and $\Phi^{\prime}$, so the new scalars have to decay into fermions. It decays almost exclusively to $t \bar{t}$ which makes it easily possible to distinguish $\Phi^{\prime}$ from $\phi^{\prime}$ experimentally.

We would like to consider as an example the heavy scalar to have a mass light enough to be produced at the LHC and the Higgs particle to have a mass of about $140 \mathrm{GeV}$. In this case the mixing cannot be neglected and the scalars decay into fermions and - although still suppressed - into gauge bosons.

The heavy neutral particle $H_{0}$ decays predominantly into fermionic channels:

$$
H_{0} \rightarrow \bar{f} f
$$

For the partial widths we find

$$
\Gamma\left(H_{0} \rightarrow \bar{f} f\right)=\tan ^{2} \theta \frac{G_{\mu} N_{c}}{2 \sqrt{2} \pi} M_{H_{0}} m_{f}^{2} \beta_{f}^{3}
$$

with $\beta=\left(1-4 m_{f}^{2} / M_{H_{0}}^{2}\right)^{1 / 2}$ being the velocity of the fermions and $N_{c}$ the color factor $N_{c}=3(1)$ for quarks (leptons).

The heavy charged scalar $H^{ \pm}$decays as follows: 


$$
H^{ \pm} \rightarrow \bar{t} b
$$

This decay channel suffers from a large irreducible background

$$
g g \rightarrow t \bar{t} g, g q \rightarrow t \bar{t} q .
$$

The partial width for $H^{ \pm}$can be calculated numerically (see [6]).

The decay widths of heavy scalars into gauge bosons are suppressed compard to the Standard Model Higgs particle by the factor $\sqrt{2}\left(M_{h} / M_{H_{0}}\right)^{4} \tan ^{2} \theta$. The partial widths for $H^{0}$ are given by

$$
\Gamma\left(H_{0} \rightarrow V V\right)=\frac{G_{\mu}}{16 \pi}\left(\frac{M_{h}}{M_{H_{0}}}\right)^{4} M_{H_{0}}^{3} \tan ^{2} \theta \delta_{V} \sqrt{1-4 x}\left(1-4 x+12 x^{2}\right), x=\frac{M_{V}^{2}}{M_{H_{0}}^{2}}
$$

with $\delta_{W}=2$ and $\delta_{Z}=1[7]$.

The largest contributions for the production cross section of a very heavy scalar are the gluon and vector boson fusion processes. The associative production with $W$ or $Z$ bosons is at least two orders of magnitude smaller for a scalar particle of more than $1 \mathrm{TeV}$.

Reduced by a factor $\arctan ^{2} \theta$ compared to a Higgs particle the gluon fusion cross section for a single $H_{0}$ can be expressed in leading order by $[8]$

$$
\sigma\left(g g \rightarrow H_{0}\right)=\tan ^{2} \theta \frac{G_{\mu} \alpha_{s}^{2}}{144 \pi}\left|\frac{3}{4} \sum_{q} A_{1 / 2}\left(\tau_{Q}\right)\right|^{2}
$$

with $\tau_{Q}=M_{H_{0}}^{2} / 4 m_{Q}^{2}$ and the form factor

$$
A_{1 / 2}\left(\tau_{Q}\right)=2\left[\tau_{Q}-\frac{1}{4}\left(\tau_{Q}-1\right)\left(\log \frac{1+\sqrt{1-\tau_{Q}^{-1}}}{1-\sqrt{1-\tau_{Q}^{-1}}}-i \pi\right)^{2}\right] .
$$

For calculation of the hadronic cross section $p p \rightarrow H_{0}$ we can use ref. 9 .

The cross section for the vector boson fusion production channel $q q \rightarrow V^{*} V^{*} q q \rightarrow H_{0} q q$ is suppressed by $\sqrt{2}\left(M_{h} / M_{H}\right)^{4} \tan ^{2} \theta$. To calculate the hadronic cross section we use the program VV2H [10].

The heavy quark associated production is a third production channel 12 . But this channel is in the here considered mass region at least two orders of magnitude smaller than the gluon fusion channel.

The only heavy scalar production channel which is not suffering from any mixing induced suppression mechanism is the scalar pair production by vector boson fusion. But this cross-section is far below $1 \mathrm{fb}$ and thus not large enough to enhance the total production cross section decisively [11.

There is also a fusion production process for the $\mathrm{H}^{+}$.

$$
u \bar{d} \rightarrow H^{+}
$$

But this process should be even smaller because the coupling between $H^{+}$and the quarks is proportional to the fermion masses. Numerical results are shown in (Table 1).

To distinguish the scalar $H_{0}$ from the pseudoscalar $H_{1}$ it is possible to use the process

$$
H_{0} / H_{1} \rightarrow t \bar{t} \rightarrow\left(W^{+} b\right)\left(W^{-} \bar{b}\right)
$$

analogously to the Higgs boson case [5].

The scalars decay almost exclusively into fermionic channels and so their detectability suffers from a large irreducible background. Especially for heavy scalar particles above $2 \mathrm{TeV}$ for which the production cross section is far below $1 f b$ it seems to be very peculiar to detect them.

But for scalars with a mass below $1 \mathrm{TeV}$ there is a good chance to find them at the LHC after collecting enough integrated luminosity.

We would like to thank D.N. Gao for valuable discussions. 


\begin{tabular}{|c||c|c|}
\hline & $M_{H_{0}}=504$ & $M_{H_{0}}=800$ \\
\hline \hline$\lambda$ & $3.5 \cdot 10^{4}$ & $5.6 \cdot 10^{4}$ \\
\hline$\Lambda$ & $11.5 \cdot 10^{4}$ & $31 \cdot 10^{4}$ \\
\hline$M_{h_{0}}$ & 140.10 & 140.04 \\
\hline$\theta$ & 15.66 & 9.93 \\
\hline$\Gamma\left(H_{0} \rightarrow \bar{t} t\right)$ & 1.74 & 2.18 \\
\hline$\Gamma\left(H^{-} \rightarrow \bar{t} b\right)$ & 1.86 & 1.36 \\
\hline$\Gamma\left(H_{0} \rightarrow W^{+} W^{-}\right)$ & $3.3 \cdot 10^{-2}$ & $8.9 \cdot 10^{-3}$ \\
\hline$\Gamma\left(H^{-} \rightarrow W^{-} Z\right)$ & $8.0 \cdot 10^{-4}$ & $2.1 \cdot 10^{-4}$ \\
\hline$\sigma\left(p p \rightarrow H_{0}\right)$ & 310 & 13 \\
\hline$\sigma\left(p p \rightarrow H_{0} q q\right)$ & $5.3 \cdot 10^{-1}$ & $1.1 \cdot 10^{-2}$ \\
\hline
\end{tabular}

Table 1: The hadronic cross sections for gluon fusion $\sigma\left(p p \rightarrow H_{0}\right)$ and for vector boson fusion $\sigma\left(p p \rightarrow q q H_{0}\right)$ are in $f b$. The partial widths and masses are in $G e V . \alpha_{s}$ was evaluated at the partonic center of mass energy.

\section{References}

[1] X. Calmet Eur. Phys. J. C, 28 (2003) 451-454 ; X.Calmet and J.F. Oliver hep-ph/0606209 (2006).

[2] R.N. Mohapatra and G. Senjanovic Phys. Rev. Lett., 44 (1980) 165; D. Chang, R.N. Mohapatra Phys. Rev.D 32 (1985) 1248.

[3] Y. Kiyo, T. Morozumi, P. Parada, M.N. Rebelo and M. Tanimoto Prog.Theor.Phys. 101(1999) 671; Z.G. Berezhiani, R.Rattazzi Phys. Lett. B, 279 (1992)124; A. Davidson, K.C. Wali Phys.Rev.Lett., 60 (1988) 1813.

[4] D. Atwood, S. Bar-Shalom and A. Soni Eur. Phys. J. C, 45 (2006) 219; S. Bar-Shalom, D.Atwood and A. Soni, PoS HEP2005 (2006) 358.

[5] M. Kramer,.J.H. Khun, M.L. Stong, P.M. Zerwas Z.Phys. C 64 (1994) 21

[6] T. Hahn, M. Perez-Victoria Comput. Phys. Commun. 118 (1999) 153.

[7] B.W. Lee, C. Quigg and H.B. Thacker Phys. Rev. D 16 (1977) 1519.

[8] H. Georgi, S. Glashow, M. Machacek and D. Nanopoulos Phys. Rev. Lett. 40 (1978) 692

[9] M. Spira Nucl.Instrum.Meth. A 389 (1997) 357

[10] K. Jakobs et al Eur.Phys.J. C 32, S2 (2004) 19.

[11] A. Djouadi, W. Kilian, M. Mühlleitner and P.M. Zerwas Eur. Phys. J. C., 45 (1999) 10

[12] J.N. Ng and P. Zakarauskas, TRI-PP-82/85 (1982). 